\title{
91 nm C+L Hybrid Distributed Raman-Erbium-Doped Fibre Amplifier for High Capacity Subsea Transmission
}

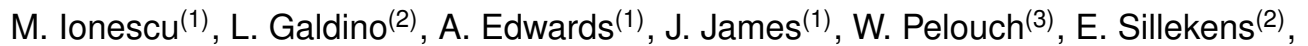 \\ D. Semrau (2), D. Lavery ${ }^{(2)}$, R. I. Killey(2), S. Barnes ${ }^{(1)}$, P. Bayvel $^{(2)}$, and S. Desbruslais ${ }^{(1)}$
}

(1) Xtera, Bates House, Church Road, Romford RM3 0SD, UK, maria.ionescu@xtera.com

(2) Optical Networks Group, University College London, Torrington Place, London WC1E 7JE, UK

(3) Xtera, 500 W Bethany Dr, Allen, TX 75013, USA

\begin{abstract}
Hybrid distributed Raman-EDFA amplifiers, with a continuous $91 \mathrm{~nm}$ gain bandwidth and $1.4 \mathrm{~dB}$ average effective noise figure, are used to enable a record single mode fibre transmission capacity of 120 Tbit/s using $312 \times 35$ GBd DP-256QAM over $9 \times 70 \mathrm{~km}$ spans.
\end{abstract}

\section{Introduction}

The consistent growth in global network traffic is driving future optical networks towards spatial and spectral domain parallelism ${ }^{1}$ to provide the required capacity. As space division multiplexed (SDM) systems are composed of parallel channels, the single mode capacity will partially dictate the total system capacity. Moreover, the relatively mature single mode transmission systems are still widely deployed, and therefore set the benchmark for what can be achieved in optical fibre communications.

Fundamental limits of an optical communication system are dominated by a combination of noise from the transceiver subsystem, optical amplifier noise, system bandwidth and optical fibre nonlinearity. Recent years have seen several advances in improving the bandwidth of optical amplifiers, and have resulted in recent experimental single-mode fibre (SMF) capacity increases.

Previous record demonstrations have relied on transmission in the $C$ and $L$ bands based on EDFA $^{2}$, distributed Raman amplification (DRA) ${ }^{3}$ or a combination of the two ${ }^{4}$, enabling up to $78 \mathrm{~nm}$ bandwidth. Employing EDFAs in each transmission window leads to a spectral gap between the two bands, whereas DRAs provide a continuous transmit bandwidth. A continuousband $100 \mathrm{~nm}$ amplifier ${ }^{5}$ was developed based on a semiconductor optical amplifier (SOA), enabling an SMF capacity record of 115.9 Tbit/s over $100 \mathrm{~km}$. Although the bandwidth is impressive, SOAs have a relatively high noise figure (NF) compared with DRAs, and so system performance decreases rapidly with distance.

In this work, we designed and tested a continuous $91 \mathrm{~nm}$ gain bandwidth hybrid distributed Raman-EDFA (HRE) as a prototype for wideband amplification that has low NF, enabling a record throughput of $120 \mathrm{~Tb} / \mathrm{s}$ over $630 \mathrm{~km}$ of SMF.

\section{Experimental configuration}

In the experimental setup, shown in Fig. 1, four carriers spaced at $35.5 \mathrm{GHz}$ were connected to two independent dual-polarization IQ optical modulators, provided by Oclaro, each driven by four $92 \mathrm{GS} / \mathrm{s}$ digital-to-analogue converters (DACs) to generate four odd/even channels. A digital root-raised cosine (RRC) filter with 0.01 roll-off was used to spectrally shape the signals and pre-emphasis was applied to overcome the electrical response of the transmitter components. The channels were generated at carrier frequencies which were tuned across the spectrum from 1524.9 to $1615.9 \mathrm{~nm}$, allowing the measurement of $312 \times 35$ GBd dual-polarization 256-ary quadrature amplitude modulation (DP-256QAM) channels.

The modulated channels were amplified using a pair of $97 \mathrm{~nm}$ bandwidth discrete Raman amplifiers with $12.5 \mathrm{~dB}$ gain and combined with wideband amplified spontaneous emission (ASE) noise, which emulated co-propagating channels over the entire transmitted bandwidth. The ASE noise, with continuity across the entire $97 \mathrm{~nm}$ bandwidth, was generated by a pair of discrete Raman amplifiers to achieve a total output power of $22 \mathrm{dBm}$ within the required $91 \mathrm{~nm}$ bandwidth, following which, a band stop filter (BSF) was used to create a notch in the ASE within which the modulated channels were positioned. The validity of using ASE noise to emulate aggressor channels was verified in ${ }^{6}$, showing that this technique provides a conservative measure of system performance.

A gain equalising amplifier (GEA) with a continuous $98 \mathrm{~nm}$ bandwidth was used to amplify 


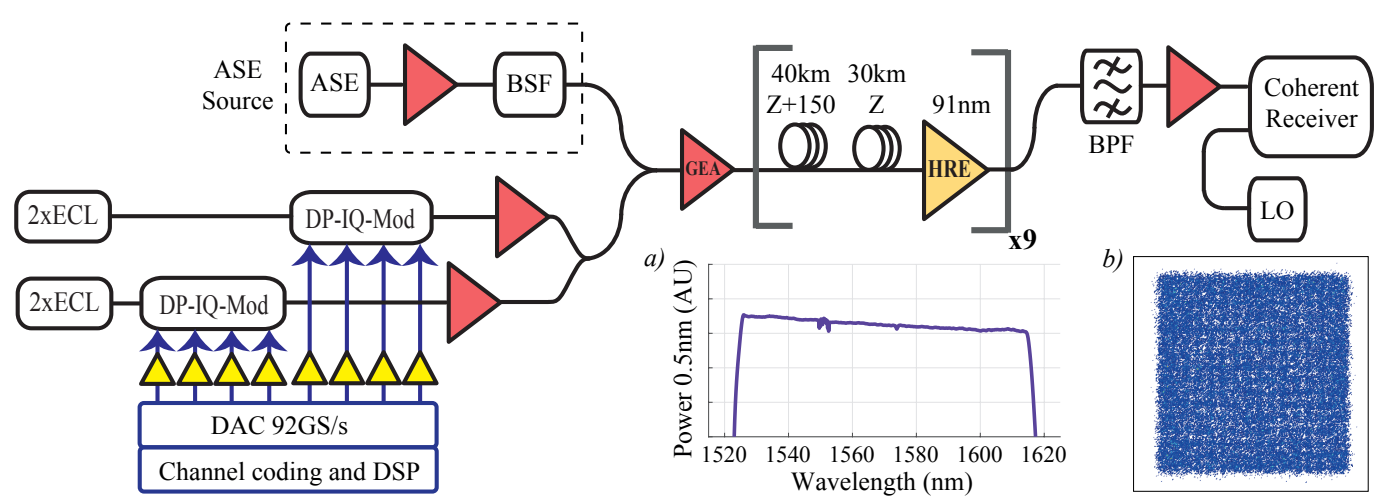

Fig. 1: Experimental setup and a) signal spectrum at the input into the first fibre span (CUI centered at $1551 \mathrm{~nm}$ ) b) 256QAM constellation in a back-to-back configuration.

and spectrally shape (SS) the combined ASE and modulated channels. The combined SS-ASE and modulated channels occupied a total usable bandwidth of $91.04 \mathrm{~nm}(11.0758 \mathrm{THz})$ with a total output power of $22 \mathrm{dBm}$. The optical spectrum after the GEA is shown as inset (a) in Fig. 1. A power tilt of $-2 \mathrm{~dB}$ across the bandwidth was applied to equalise the channel performance, taking into account the wavelength-dependent NF of the transmission line amplifiers. The back-to-back 256QAM constellation is illustrated in the inset (b) of Fig. 1.

The transmission link comprised a straight-line of 9 spans, with $70 \mathrm{~km}$ of SMF and an HRE in each span. Each fibre span mixed two fibre types: $40 \mathrm{~km}$ of Sumitomo $Z+150$ fibre with an average attenuation of $0.148 \mathrm{~dB} / \mathrm{km}$ and an effective core area of $149 \mu \mathrm{m}^{2}$, followed by $30 \mathrm{~km}$ of Sumitomo $Z$ fibre with an average attenuation of $0.16 \mathrm{~dB} / \mathrm{km}$ and an effective core area $81 \mu \mathrm{m}^{2}$. The $Z+150$ fibre reduces the nonlinear penalties on the transmit side of the span while the $Z$ fibre provides sufficient Raman gain to achieve $0 \mathrm{~dB}$ net gain across the spectrum.

Each HRE provided continuous gain from $1525 \mathrm{~nm}$ to $1616 \mathrm{~nm}$ and used two counterpropagating pumps at 1427 and $1495 \mathrm{~nm}$, as shown in Fig. 2, with output powers of $300 \mathrm{~mW}$ and $310 \mathrm{~mW}$ into the transmission fibre, delivering a total power of $19.5 \mathrm{dBm}$ to the EDFA stage. The single EDFA stage boosted the signal to a total output power of $22 \mathrm{dBm}$, and included a $91 \mathrm{~nm}$ gain flattening filter (GFF) to equalise the gain across the entire HRE bandwidth. The distributed amplification approach reduces the NF compared to EDFA only or SOA techniques. As shown in Fig. 3 the effective NF was $1.4 \mathrm{~dB}$ on average.

At the receiver, the average signal-to-noise ratio (SNR) across the bandwidth was $18.9 \mathrm{~dB}$. Since the back-to-back SNR was approximately

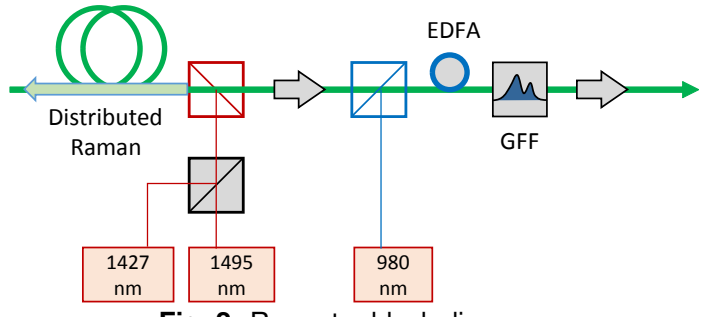

Fig. 2: Repeater block diagram.

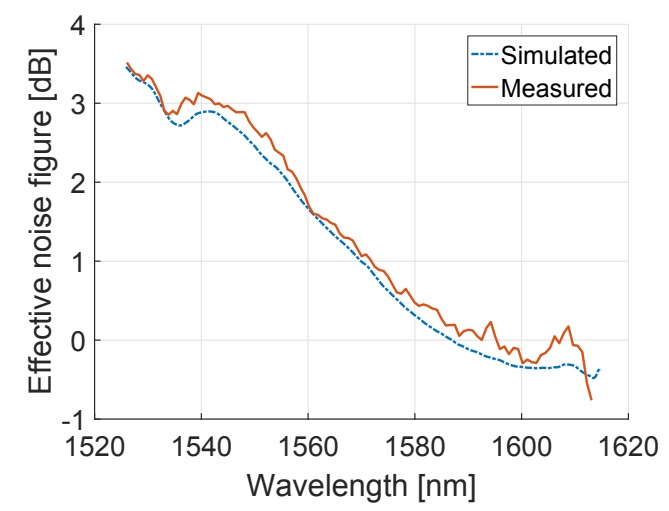

Fig. 3: Effective noise figure of an HRE prototype.

$21 \mathrm{~dB}$, this suggests that the SNR degradation is dominated by the transceiver performance and the impact of the HRE ASE is minimal. A bandpass filter (BPF) with a $40 \mathrm{GHz}$ bandwidth was used to filter the channel under test (channel 2 of 4 ) and exclude the out-of-band noise from the receiver. A phase- and polarization-diverse coherent receiver incorporating $70 \mathrm{GHz}$ bandwidth photodetectors was employed, and the signal was digitised using a real-time oscilloscope with $63 \mathrm{GHz}$ bandwidth, sampling at $160 \mathrm{GSa} / \mathrm{s}$. Digital signal processing was performed as described in ${ }^{6}$, which included matched filtering, single step chromatic dispersion compensation, a 21-tap blind adaptive equaliser, frequency offset compensation and decision directed carrier phase estimation. The system performance was quantified in terms of throughput and BER, after decoding with soft-decision forward error correction (SD-FEC). 


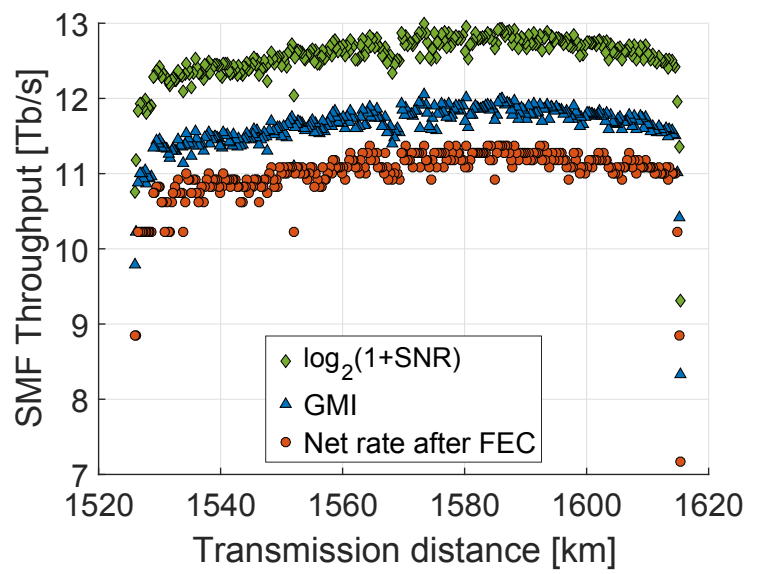

Fig. 4: Throughput per channel over 2 polarizations after $630 \mathrm{~km}$.

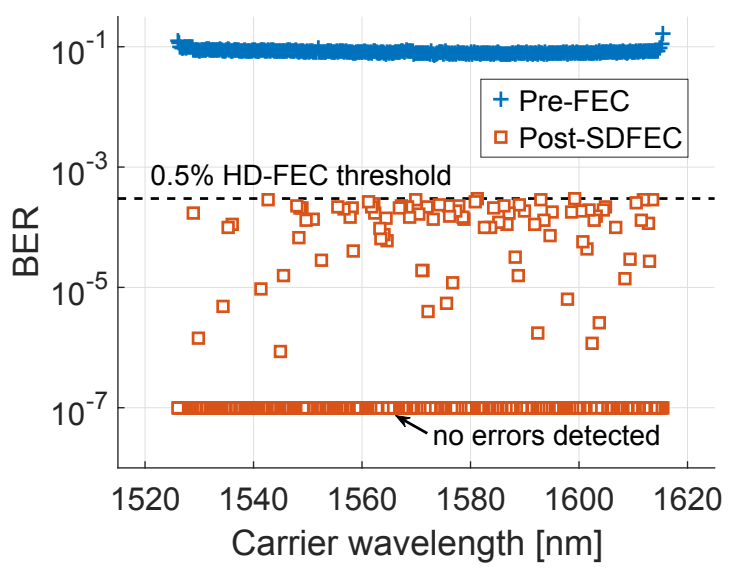

Fig. 5: Pre-FEC and post-SD-FEC BER for all 312 channels.

\section{Results}

The information rate of all $312 \times 35 \mathrm{GBd}$ DP256QAM channels was calculated over the continuous $91 \mathrm{~nm}$ bandwidth and is shown in Fig.4. The Shannon limit for the received SNR is given by $\log _{2}(1+\mathrm{SNR})$ summed over both polarizations. The total received SNR was evaluated as the ratio between the variance of the transmitted symbols $E\left[|X|^{2}\right]$ and the variance of the noise $\sigma^{2}$, where $\sigma^{2}=E\left[|X-Y|^{2}\right]$ and $Y$ represents the received symbols. The pre-FEC GMI was calculated using received log-likelihood ratios. A mean penalty of $0.94 \mathrm{bit} / \mathrm{symbol}$ between the GMI (11.62 bit/symbol) and the Shannon limit (12.56 bit/symbol) is due to the use of non-optimal finite constellation and bit labeling. For the net rate after inner and outer FEC, the occupied spectrum yields a net bit rate of 10.99 bits/symbol providing a record single mode fibre capacity of $120.0 \mathrm{Tbit} / \mathrm{s}$.

Fig. 5 shows the pre- and post-SD-FEC BER for all 312 channels. The channels were decoded using 12 rate adapted LDPC codes implemented from the DVB-S2x standard. An outer BCH HDFEC code with a bit error rate (BER) threshold of
$3 \times 10^{-4}$ with $0.5 \%$ overhead was assumed. All 312 channels were measured, confirming the total net throughput of $120.0 \mathrm{Tbit} / \mathrm{s}$. The bulk of the codes have a low spread in code rate, 293 out of 312 have between $40-50 \%$ FEC overhead. Reducing the number of code rates applied to 7 was found to reduce the total net data throughput to $119 \mathrm{Tbit} / \mathrm{s}$.

\section{Conclusions}

This work demonstrated a prototype widebandwidth hybrid EDFA-Raman amplifier for transmission over SMF. This technique offers distributed gain, and so inherently maintains a low noise figure and a high SNR over longer transmission distances, compared to previously proposed solutions. In contrast to EDFA-based $\mathrm{C}+\mathrm{L}$ band transmission systems, the continuous $91 \mathrm{~nm}$ gain bandwidth offered by hybrid amplification means that no spectrum is wasted.

We demonstrated record throughput of 120 Tbit/s transmitted over $630 \mathrm{~km}$ of SMF, an improvement over the previous capacity record ${ }^{5}$, with an extra 4 Tbit/s capacity over more than 6 times longer transmission distance.

\section{Acknowledgements}

The authors gratefully acknowledge the support of UK EPSRC UNLOC, INSIGHT and Royal Academy of Engineering Research Fellowships, Oclaro for the modulators and Nick Hepden for the heroic effort in constructing the hybrid amplifiers used in this work.

\section{References}

[1] P. J. Winzer and D. T. Neilson, "From Scaling Disparities to Integrated Parallelism: A Decathlon for a Decade," JLT, vol. 35, no. 5, pp. 1099-1115, March, 1 (2017).

[2] T-X. Cai et al., "70.46 Tb/s over 7,600 km in in C+ L Band Using Coded Modulation with Hybrid Constellation Shaping and Nonlinearity Compensation," Proc. OFC, PDP Th5B.2 (2017).

[3] L. E. Nelson, et. al., "All-Raman-Amplified, $73 \mathrm{~nm}$ Seamless Band Transmission of $9 \mathrm{~Tb} / \mathrm{s}$ Over $6000 \mathrm{~km}$ of Fiber," in PTL, vol. 26, no. 3, pp. 242-245, Feb.1, (2014).

[4] L. Krzczanowicz, et. al., "Hybrid discrete Raman/EDFA design for broadband optical amplification in metro WDM systems," 19th ICTON, pp. 1-4 (2017).

[5] J. Renaudier et al., "First 100-nm Continuous-Band WDM Transmission System with 115Tb/s Transport over 100km Using Novel Ultra-Wideband Semiconductor Optical Amplifiers," Proc. ECOC, PDP (2017).

[6] D. J. Elson et al., "Investigation of Bandwidth Loading in Optical Fiber Transmission using Amplified Spontaneous Emission Noise," Optics Express 25 (16), (2017). 